\title{
Study of an asynchronously rotating polar CD Ind*
}

\author{
Arti Joshi ${ }^{\dagger}$, Jeewan Chandra Pandey
}

Aryabhatta Research Institute of observational sciencES, Manora Peak, Nainital 263 001, India

\begin{abstract}
We present X-ray and ultaviolet (UV) analyses of the magnetic cataclysmic variable CD Ind. The $\mathrm{X}$-ray light curves obtained from different observations show a very chaotic and spectacular pattern. X-ray and UV variations occur at orbital periods of $(124.0 \pm 0.4)$ and (112.3 \pm 0.4$) \mathrm{min}$ and spin periods of (119.4 \pm 0.4$)$ and (109.8 \pm 0.3$) \mathrm{min}$, respectively. The degree of asynchronism of CD Ind is calculated as $\sim 2.2 \%$ and $\sim 3.7 \%$ from the UV and X-ray data, respectively. The variable shape of the X-ray and UV light curves indicate that the geometry of accretion changes in every epoch of observations. A two-pole accretion mode geometry is also visible in the X-ray and UV light curves of CD Ind. This two-pole accretion geometry is supported by the presence of double humps or dips in their X-ray and UV light curves. The rotational modulations of UV data are also found to be anti-correlated with respect to the X-rays, revealing that the UV pulsations are not originating in the region of magnetically confined accretion flow. Unlike other magnetic CVs, a strong fluorescent $\mathrm{Ni} \mathrm{K} \alpha$ emission line is detected for the first time in the X-ray spectra of CD Ind. The X-ray and UV variations of CD Ind support the view that this system belongs to the class of asynchronous polars.
\end{abstract}

Keywords: accretion, — binaries: close — magnetic CV, cataclysmic variables — stars: individual (CD Ind) - X-rays: stars

\section{Introduction}

Polars are a class of magnetic cataclysmic variables $(\mathrm{CVs})$ in which material transfers from the Roche lobefilling red dwarf companion to the magnetized white dwarf (WD) primary. These binary systems are synchronously locked ( $\mathrm{P}_{\omega}=\mathrm{P}_{\Omega}$, where $\mathrm{P}_{\Omega}$ and $\mathrm{P}_{\omega}$ are the orbital and spin periods, respectively) with the high magnetic field $(\mathrm{B} \geq 10 \mathrm{MG})$ of the WD. The high magnetic field strength in the polars causes the accretion stream to flow along the magnetic field lines to its poles and suppresses the formation of the accretion disc leading to a synchronous motion. These magnetically channelled accretion stream forms a shock above the WD surface. Below the post-shock region, the material cools via thermal bremsstrahlung and cyclotron radiation (Aizu 1973, Wu 1994, Cropper 1999), depending upon the magnetic field strength of the WD, and produces hard and soft X-rays. In polars, cyclotron cooling is more efficient (Lamb 1979, Woelk 1992). Therefore, a part of the downward emitted hard X-rays and cyclotron radiation is absorbed by the WD surface and re-emitted in soft X-rays or in the extreme UV. The soft X-ray photons are mostly emitted near the base of the post-shock flow. The majority of polars also show a soft X-ray excess. Various predictions have been put forward to account for the soft X-ray excess. The most widely accepted solution was proposed by Kuijpers et al. (1982) and further developed by Frank et al. (1988). Kuijpers et al. (1982) suggest that the soft-X-ray excess could be produced by dense blobs of material which penetrate into the WD photosphere and the energy is thermalized to a black-body. However, some intermediate polars (IPs) (PQ Gem, NY Lup, V2400 Oph, etc.) also possess a

\footnotetext{
${ }^{*}$ An Asynchrnous Polar

†arti@aries.res.in, aartijoshiphysics@gmail.com
} 
soft X-ray emission component (Mason et al. 1992, Haberl et al. 1994, Joshi et al. 2019) which are referred to as 'soft-IPs'.

There are some other magnetic CVs like V1432 Aql (Friedrich 1996), BY Cam (Silber et al. 1997, Mason et al. 1998), V1500 Cyg (Katz et al. 1991), and CD Ind (Schwope et al. 1997) which are described as asynchronous polars (APs) for which the spin and orbital periods differ by about $2 \%$ or less. Recently, RX J0838-2827 was also found to be an asynchronous polar by Rea et al. (2017). These APs are thought to arise due to a nova explosion in the past. It is also believed that they will return to synchronism in hundreds of years (Boyd et al. 2014). Besides these systems, the sources Paloma (Joshi et al. 2016) and IGR J19552+0044 (Bernardini et al. 2013) were also found to be asynchronous, but they have a large asynchronicity compared to the above systems. Among these four confirmed APs, CD Ind is the only AP which lies below the 2-3 hour period gap. This source is detected during the EUVE all-sky survey (Bowyer et al. 1996) and ROSAT X-ray all-sky survey (Voges et al. 1996). Ramsay et al. (1999) observed positive and negative circular polarization humps and inferred that the accretion occurs at two different regions in this system. There is no distinct soft Xray component seen in CD Ind because the reprocessed component is cooler and shifted to extreme ultraviolet (EUV) or UV energies (Ramsay et al. 2002). Schwope et al. (1997) determined the magnetic field strength for this system in the range of $9-13$ MG. Therefore, this system is also described as 'low-field polar', similar to the polars AM Her and V2301 Oph.

In this paper, we present detailed X-ray and UV analyses of CD Ind. This paper is organized as follows. In section 2, we describe the observational data sets that we have analysed and the methods of data reduction. Section 3 contains the analysis and results of the X-ray and UV observations, including timing analysis as well as spectral analysis. Finally, we present a discussion in section 4.

\section{Observations and Data Reduction}

CD Ind was observed with the XMM-Newton satellite (Jansen et al. 2001) using the European Photon Imaging Camera (EPIC; Struder et al. 2001, Turner et al. 2001) from 27 March 2002 to 2 April 2002 with seven observation IDs. The EPIC instrument consists of three cameras containing the MOS1 and MOS2 (Turner et al. 2001) and PN (Struder et al. 2001) CCDs, providing extremely sensitive imaging observations over a field of view of $30 \mathrm{arcmin}$ in an energy range of $0.2-12.0 \mathrm{keV}$. We used the standard XMM-Newton Science Analysis System (SAS) software package (version-17.0.0) for data reduction with updated calibration files. The preliminary processing of raw EPIC Observation Data Files (ODF) was done using the EPCHAIN and EMCHAIN tasks. It allows to calibrate the events registered in each CCD chip both in energy and astrometry and to combine them in a single data file for MOS and PN detectors. The background contribution is particularly relevant at high energies. Therefore, for further analysis, we have selected an energy range between 0.3 and $10.0 \mathrm{keV}$. Calibrated and concatenated event list files were extracted using the SAS task EVSELECT. Data were corrected for the barycenter of the solar system with the BARYCEN task. We have also checked the data for high background proton flares but no such events were detected. Subsequently, the EPATPLOT task was used for checking the existence of pile up but all observations were free of this effect. The X-ray light curves and spectra of CD Ind were extracted from a circular region of radius 40 arcsec around the source. The background was calculated from several source-free regions on the detectors surrounding the source, each with the same radius of 40 arcsec. Further, the SAS task EREGIONANALYSE was used for the final region selection. Good time intervals, dead time, exposure, point-spread function, quantum efficiency, and background subtraction are corrected by using the task EPICLCCORR. The SAS task ESPECGET was used to generate the background corrected spectra, which also computes the photon redistribution matrix and the ancillary matrix. The spectra were rebinned for a minimum of 20 counts per bin using GRPPHA. Further temporal and spectral analyses were done with standard packages HEASOFT version-6.24 and XSPEC version-12.9.0.

CD Ind was also observed with the Optical Monitor instrument (OM; Mason et al. 2001) in the UVW1 filter with an effective wavelength of $291 \mathrm{~nm}$. This provides a facility to study the source simultaneously in UV and X-ray bands. All observations were carried out in fast imaging mode for a total exposure time of $86.4 \mathrm{ks}$. The OM fast imaging mode data were reprocessed with the standard reduction pipelines included in SAS. 

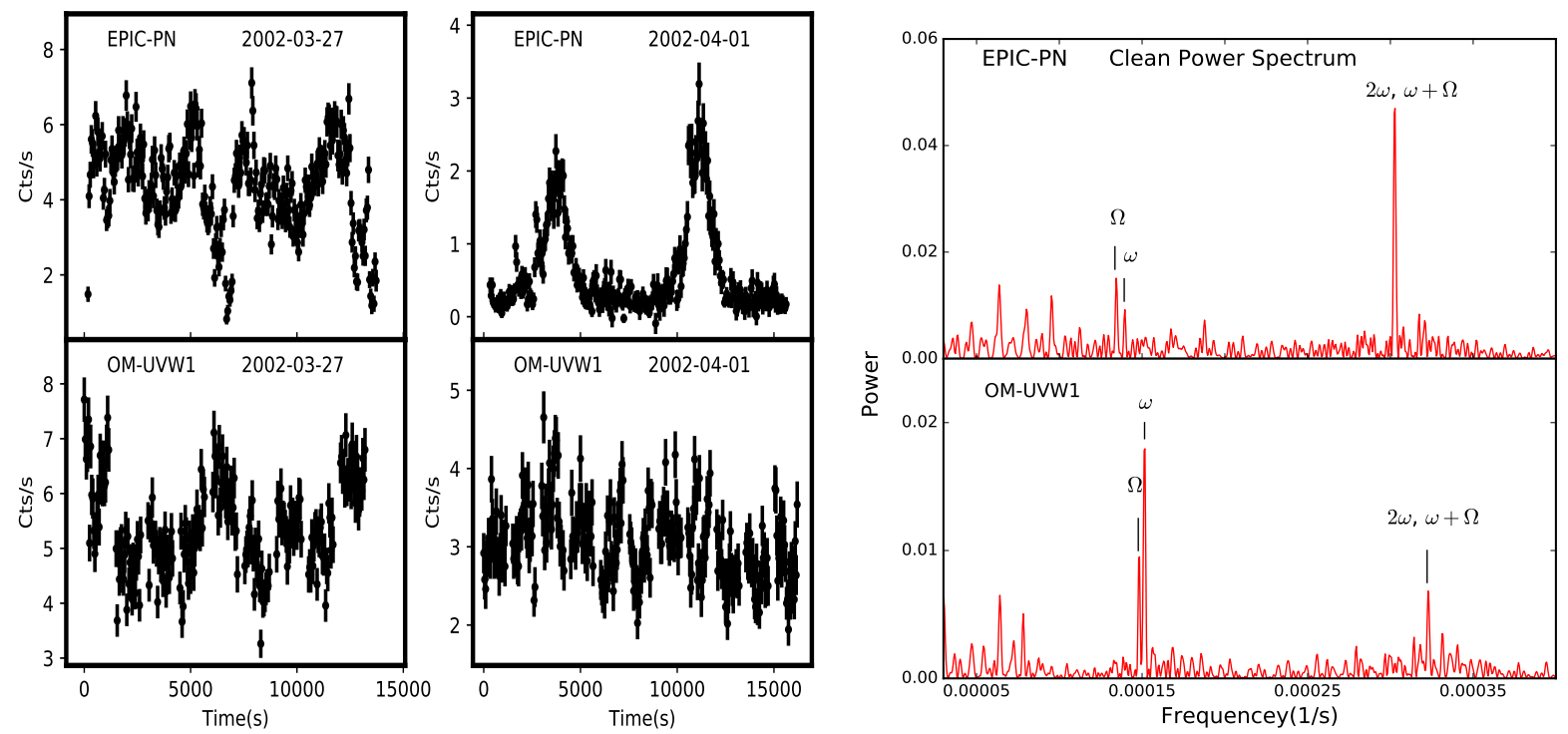

Figure 1: X-ray (top) and UV (bottom) light curves of CD Ind. In each panel, the observation date is also mentioned.

Figure 2: CLEANed X-ray (top) and UV (bottom) power spectrum of CD Ind.

\section{Analysis and Results}

\subsection{Light Curves and Power Spectra}

In Fig. 1, the background-subtracted X-ray and UV light curves of CD Ind with a $50 \mathrm{~s}$ binning are shown for epochs 27 March 2002 (left) and 1 April 2002 (right). The X-ray light curves show an irregular pattern for each epoch of observations. The periodicities in the X-ray and UV light curves of CD Ind were searched by using the CLEAN algorithm (Roberts et al. 1987) in the timing data. The CLEANed power spectrum of X-ray and UV light curves are shown in the top and bottom panels of Fig. 2, respectively. The significant periods were derived as $(124.0 \pm 0.4)$ and $(119.4 \pm 0.4) \mathrm{min}$ from the EPIC-PN observations, while $(112.3 \pm 0.4)$ and $(109.8 \pm 0.3)$ min is found from the OM-UVW1 observations. The periods determined from the X-ray data are found to be consistent with the periods reported by Schwope et al. (1997). However, the periods derived by OM-UVW1 data closely match with the periods determined recently by Myers et al. (2017) and Littlefield et al. (2019) using large data sets of photometric observations. These periods appear to be the orbital and spin periods of CD Ind. For the X-ray and UV observations, the other dominant peak in the power spectra corresponds to the frequency of $\omega+\Omega$ (or $2 \omega)$ at (55.0 \pm 0.1$)$ and (51.6 \pm 0.1$)$ min, respectively.

We have also folded the X-ray and UV light curves for both above-described epochs using the ephemeris as given by Myers et al. (2017). The folded X-ray light curves corresponding to the derived orbital and spin periods are shown in top panels of Fig. 3 and Fig. 4, respectively. Strong orbital and spin modulations are detected in X-ray bands. On 27 March 2002, two dips are clearly visible in both orbital and spin phase folded $\mathrm{X}$-ray light curves. In these light curves, the second dip was found to be more prominent compared to the first one. Besides this eclipse like structure, on 1 April 2002, a clear single hump-like structure is seen in the orbital phase folded X-ray light curves near phase $\sim 0.5$. However, the spin modulations appears to be maximum around phase $\sim 0.0$. The orbital and spin phase folded UV light curves are also shown in the middle panels of Fig. 3 and Fig. 4, respectively. Similar to the X-ray light curves, the double hump-like structure is also seen in the UV light curves of CD Ind for both epochs of observations, except during the binary motion of the epoch 1 April 2002. These UV modulations are found to be anti-correlated with respect to the X-rays. The folded hardness ratio (HR) curves corresponding to the derived orbital and spin periods are shown in the bottom panels of Fig. 3 and Fig. 4, respectively. The HR is estimated as $(\mathrm{H}-\mathrm{S}) /(\mathrm{H}+\mathrm{S})$, where $\mathrm{H}$ and $\mathrm{S}$ are count rates in the $2.0-10.0 \mathrm{keV}$ and $0.3-2.0 \mathrm{keV}$ energy bands, respectively. Orbital and spin period modulations are also clearly 

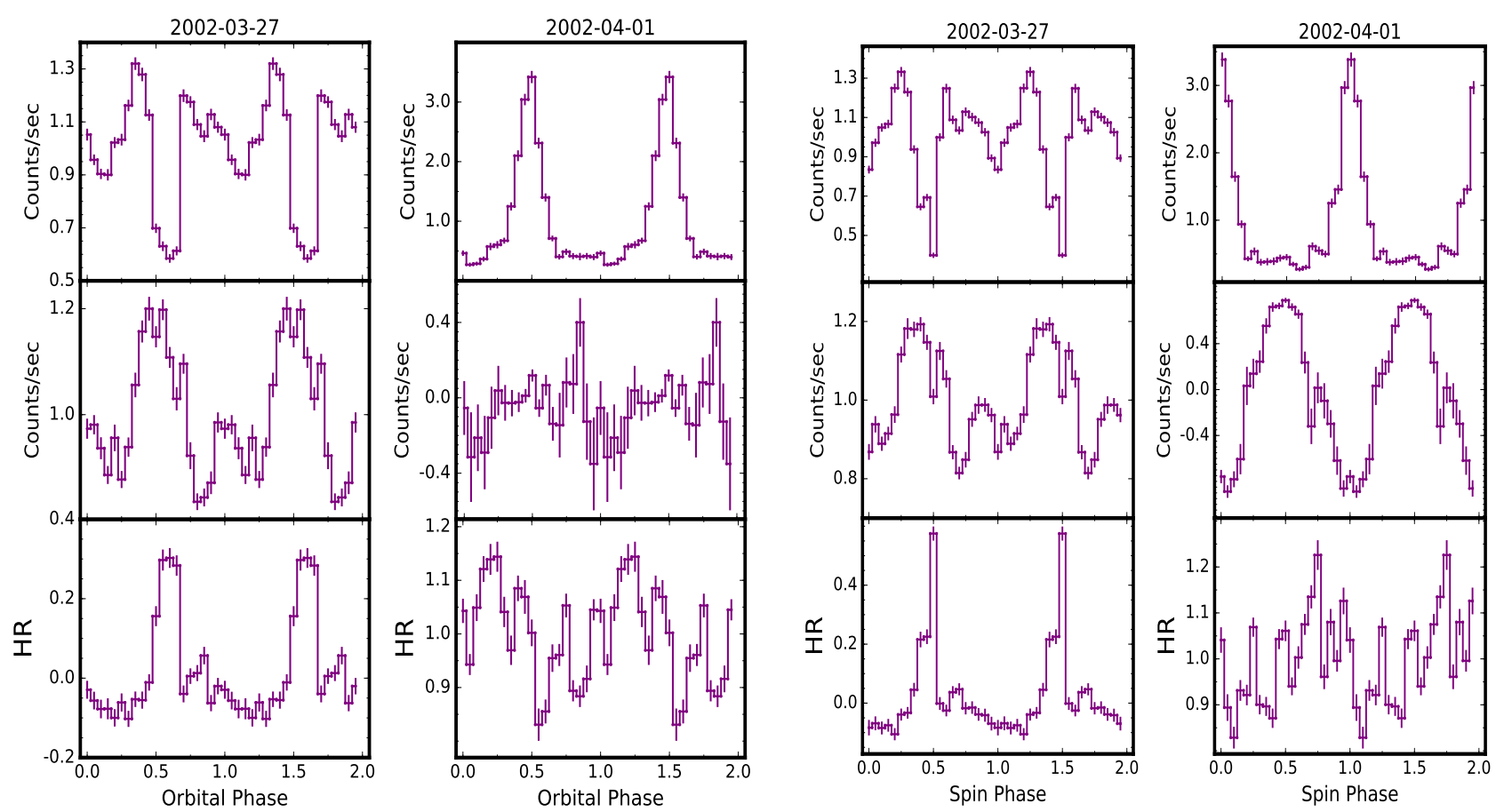

Figure 3: The $\mathrm{X}$-ray light curves in the $0.3-10.0 \mathrm{keV}$ energy range (top), the UV light curves (middle), and the hardness ratio (HR) curves extracted from the soft and hard X-ray count rates (bottom) phase folded with the orbital period. In top panels, the observation dates are also mentioned.

Figure 4: The X-ray light curves in the $0.3-10.0 \mathrm{keV}$ energy range (top), the UV light curves (middle), and the hardness ratio (HR) curves extracted from the soft and hard X-ray count rates (bottom) phase folded with the spin period. In top panels, the observation dates are also mentioned.

seen in the HR curves. There is an anti-correlation between the variations in X-ray intensities and the hardness ratio curves, both in the orbital and spin phases.

We have also explored the periodic variability at different energies. Light curves of X-ray observations for epochs 27 March 2002 and 1 April 2002 were extracted in the four energy bands: $0.3-1.0 \mathrm{keV}, 1.0-2.0 \mathrm{keV}$, $2.0-5.0 \mathrm{keV}$, and $5.0-10.0 \mathrm{keV}$. These extracted light curves were folded with the derived orbital and spin periods using the same ephemeris as given by Myers et al. (2017). Strong spin and orbital modulations are detected from soft to hard X-ray energy bands. On the epoch 27 March 2002, the amplitude of the eclipse appears to be changed from soft to hard X-ray energy bands. In this epoch, the second dip was found to be more prominent in soft X-rays, while moving towards the hard X-rays the first dip looks to be more pronounced. The lowest part of eclipse also changes with the energies from soft to hard X-rays. The morphology of the bright hump-like structure for the epoch 1 April 2002 is also seen to be energy dependent. In this epoch, the pulse counts appear to increase towards hard X-ray energies.

\subsection{X-ray Spectra}

The background-subtracted X-ray spectra of CD Ind show a strong broad band continuum along with the emission lines. The detected strong emission line near $7.4 \mathrm{keV}$ is identified as a $\mathrm{Ni} \mathrm{K} \alpha$ emission line. In general, the iron fluorescent emission lines are visible in the X-ray spectra of magnetic CVs. However, the first detection of the Ni K $\alpha$ emission was reported by Sako et al. (1999) in ASCA spectra of X-ray binaries. In case of magnetic $\mathrm{CVs}$, this is the first time that a clear evidence for the $\mathrm{Ni} \mathrm{K} \alpha$ emission line has been found in the X-ray spectra of CD Ind.

The X-ray spectral analysis of CD Ind was performed by using XSPEC (Arnaud et al. 1996, Dorman 


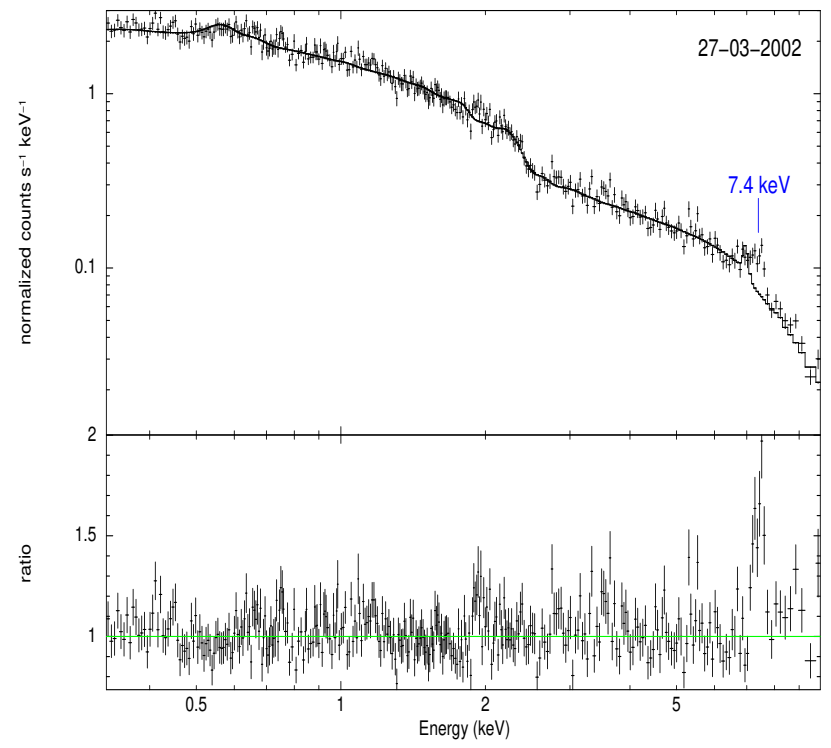

Figure 5: The best-fit X-ray spectra of CDInd for epoch 27 March 2002 without a Gaussian line component (top), and the ratio of the data to the best-fit model (bottom).

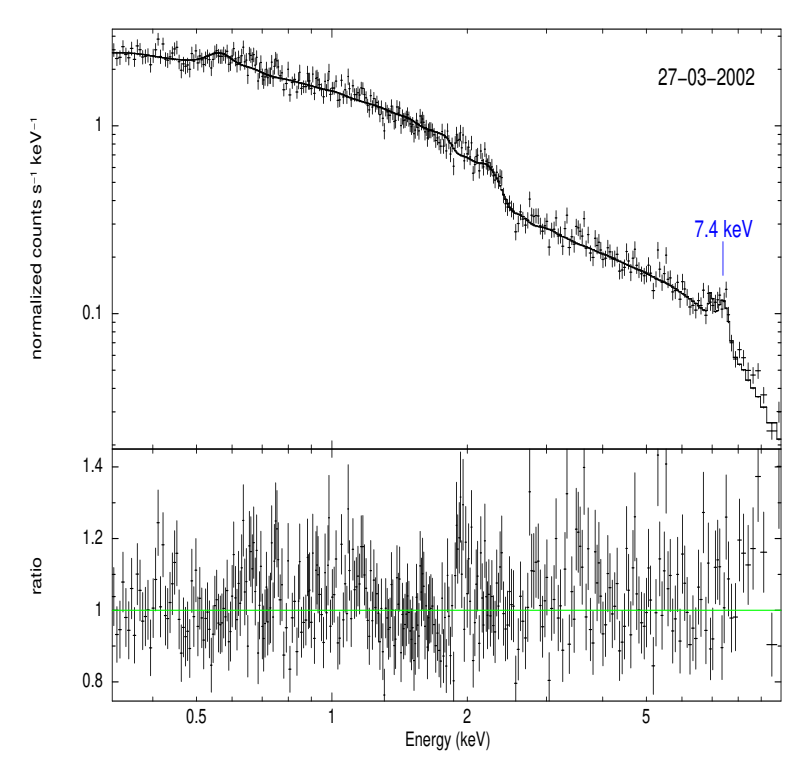

Figure 6: The best-fit X-ray spectra of CD Ind for epoch 27 March 2002 with a Gaussian line component (top), and the ratio of the data to the best-fit model (bottom).

et al. 2001) with the various models/combinations of models along with a common absorption component (TBABS; Wilms et al. 2000), which account for absorption by the neutral interstellar matter. The amount of hydrogen column density of the total absorber was found to be very low (close to zero) compared to Dickey \& Lockman (1990) for both observations. The value of this badly constrained component had no effect on the other parameters, so it was not required for further spectral fitting. Therefore, we did not find any sign of Galactic absorption in the X-ray spectra of CD Ind. Further, a different approach was taken by including the partially covering absorption model (PCFABS) with the two-temperature plasma emission model, which resulted in a high value of $\chi^{2}(>1.2)$. We also noticed fluctuating residuals around the $7.4 \mathrm{keV}$ region (Fig. 5, bottom panel). In order to reduce the high sigma deviations in the residuals near $7.4 \mathrm{keV}$, a Gaussian shaped line centered at $7.4 \mathrm{keV}$ has been taken into account, resulting in a satisfactory fit $\left(\chi_{\nu}^{2}=1.1\right)$ and residuals free of excess emission near $7.4 \mathrm{keV}$ (Fig. 6). In contrast to the epoch 27 March 2002, the spectra of the epoch 1 April 2002 have poor count statistics and are best-fitted with the two-temperature plasma emission model and one Gaussian component. The galactic hydrogen column density corrected flux $\left(f_{X}\right)$ was calculated by using the CFLUX model in the energy band $0.3-10.0 \mathrm{keV}$ for both observations. All the best-fit parameters of the continuum and spectral lines for both observations are given in Table 1, where the quoted error bars correspond to a $90 \%$ confidence limit for a single variable parameter.

\section{Discussion}

We have carried out X-ray and UV analyses of the magnetic CV CD Ind. The chaotic and spectacular light curves of CD Ind show streams of different magnetic orientations. We detected significant periods at (124.0 \pm $0.4)$ and $(119.4 \pm 0.4) \mathrm{min}$ from X-ray data and (112.3 \pm 0.4$)$ and $(109.8 \pm 0.3) \mathrm{min}$ from UV data. They appear to be the orbital and spin periods of CD Ind. In a previous study, Vennes et al. (1996) derived orbital periods of $110.8 \mathrm{~min}$ or $102.8 \mathrm{~min}$ for CD Ind from the spectroscopic observations. Later, Schwope et al. 
Table 1: Best-fit values of the X-ray spectral parameters for the two epochs of observations of CD Ind, where $N_{H}$ is partial covering absorber density (i.e. the absorption due to the partial covering of the $\mathrm{X}$-ray source by the neutral hydrogen column; in $\mathrm{cm}^{-2}$ ), $p c f$ is the covering fraction of the partial absorber, $k T_{1}$ and $k T_{2}$ are the MEKAL temperatures (in $\mathrm{keV}$ ), $n_{1}$ and $n_{2}$ are the normalization constants of MEKAL, $E$ is the central energy of $\mathrm{Ni} \mathrm{K} \alpha$ emission line (in $\mathrm{keV}$ ), $E W$ is the equivalent width of the $\mathrm{Ni} \mathrm{K} \alpha$ emission line (in eV), $f_{g}$ is the line flux of $\mathrm{Ni} \mathrm{K} \alpha$ (in photons $\mathrm{cm}^{-2} \mathrm{~s}^{-1}$ ), and $f_{X}$ is the unabsorbed X-ray flux (in erg $\mathrm{cm}^{-2} \mathrm{~s}^{-1}$ ).

\begin{tabular}{|c|cc|}
\hline Parameters & \multicolumn{2}{|c|}{ Epoch } \\
& $27-03-2002$ & $01-04-2002$ \\
\hline$N_{H}\left(\times 10^{23}\right)$ & $4_{-3}^{+2}$ &.. \\
$p c f(\%)$ & $25_{-8}^{+14}$ &.. \\
$k T_{1}$ & $0.12_{-0.01}^{+0.02}$ & $1.10_{-0.10}^{+0.12}$ \\
$n_{1}\left(\times 10^{-3}\right)$ & $0.44_{-0.12}^{+0.20}$ & $0.04_{-0.01}^{+0.01}$ \\
$k T_{2}$ & $<80$ & $19_{-5}^{+9}$ \\
$n_{2}\left(\times 10^{-2}\right)$ & $1.20_{-0.13}^{+0.30}$ & $0.11_{-0.05}^{+0.05}$ \\
$E$ & $7.42_{-0.04}^{+0.05}$ & $7.48_{-0.12}^{+0.13}$ \\
$E W$ & $159_{-38}^{+45}$ & $248_{-53}^{+133}$ \\
$f_{g}\left(\times 10^{-5}\right)$ & $5.00_{-1.20}^{+2.00}$ & $1.30_{-0.50}^{+0.50}$ \\
$f_{X}\left(\times 10^{-11}\right)$ & $1.78_{-0.02}^{+0.02}$ & $0.20_{-0.01}^{+0.01}$ \\
$\chi_{\nu}^{2}(\mathrm{DOF})$ & $1.1(946)$ & $1.2(338)$ \\
\hline
\end{tabular}

(1997) found the highest peak to correspond to a period of $109.84 \mathrm{~min}, 113 \mathrm{~min}, 114 \mathrm{~min}$, and $131 \mathrm{~min}$ using photometric, spectroscopic, and polarimetric data, respectively. They also reported that the pre-whitened CCD data analyses suggests an orbital period of $124 \mathrm{~min}$. Further, Ramsay et al. (1999) estimated that the two highest peaks correspond to periods of $110.8 \mathrm{~min}$ and $109.54 \mathrm{~min}$ using optical polarimetric data. After that, Myers et al. (2017) estimated an orbital period of $110.82 \mathrm{~min}$ and a WD spin period of $109.65 \mathrm{~min}$ using a large data set of photometeric observations of CD Ind. Recently, Littlefield et al. (2019) derived the orbital and spin periods of CD Ind as $112 \mathrm{~min}$ and $110.8 \mathrm{~min}$, respectively, using Transiting Exoplanet Survey Satellite (TESS) photometry and Doppler tomography. The determination of the exact orbital and spin periods of nearsynchronous or asynchronous polars is not so trivial. Asynchronous polars provide a unique opportunity to study the coupling of accretion streams to the magnetic field, as the orientation of the magnetic field relative to the secondary changes slowly over different time scales. Due to this, the accretion geometry changes including magnetic pole-switching of the flow during the beat cycle. Thus, if the accreting material participates in the pole-flipping during the spin-orbital beat cycle, different periods can be obtained from observations covering different fractions of the beat cycle (Mouchet et al. 1997). Apart from the orbital frequency $(\Omega)$ and the spin frequency $(\omega)$, the detection of a signal at frequency $2 \omega$ also implies that there are two accreting spots, almost opposite to each other.

Magnetic CVs occupy a wide range of parameter space in the $\mathrm{P}_{\omega}-\mathrm{P}_{\Omega}$ plane in which the evolution of these systems can be derived from the degree of synchronization with the orbital period. Similar to the majority of polars, the location of CD Ind was found below the 2-3 hr period gap (Ferrario et al. 2015), which indicates that $\mathrm{CD}$ Ind is close to, but not right at, synchronism. From the spin-to-orbit period ratio, the degree of asynchronism of CD Ind is calculated as $\sim 2.2 \%$ and $\sim 3.7 \%$ from the UV and X-ray data, respectively. Furthermore, the values of $P_{\omega} / P_{\Omega}(\sim 0.97$ or 0.96$)$ obtained from X-ray and UV analyses of CD Ind satisfy the synchronization condition $P_{\omega} / P_{\Omega}>0.6$ as derived by Norton et al. (2004). The observed asynchronicity in CD Ind suggests that this system is close to attaining synchronism and that it is evolving into the pure class of polars.

The two pole accretion mode geometry is further confirmed by the X-ray and UV light curves of CD Ind during its rotation and binary motion. In the X-ray light curves of 27 March 2002 two dips are clearly observed 
in which one dip is found to be more prominent compared to the other, which suggests that the pole accreting more matter is mostly occulted as the WD rotates. In contrast to this epoch, a prominent single-peaked light curve is observed on 1 April 2002 during the binary motion indicating that only one pole is visible, i.e. the second pole is "turned off" and the brightness is not coming from the second pole during the binary motion. However, two prominent maxima are observed during the rotational motion further revealing that accretion occurs from both poles at the same rate. Similar to X-ray data, the UV variations also show two hump-like structures that appear to be anti-correlated with the X-rays, revealing that the UV pulsations are not originating from the region of magnetically confined accretion flow but may be arising due to the heating of a larger area on the WD surface. In addition to the X-ray and UV modulations, the variations in the HR curve are found to be maximum near the spin minimum. It indicates that the strong absorption of X-rays is due to the presence of additional absorbers in an impact site of the accretion curtain.

Unlike other magnetic $\mathrm{CVs}$, we found evidence for the first time for a strong $\mathrm{Ni} \mathrm{K} \alpha$ emission line in the $\mathrm{X}$-ray spectrum of CD Ind, while there is no detection of the $\mathrm{Fe} \mathrm{K} \alpha$ emission line. The detection of the Ni $\mathrm{K} \alpha$ emission line in the $\mathrm{X}$-ray spectra of CD Ind suggests that most of the nickel is present in the region where the line is produced. Fluorescent emission from externally X-ray-illuminated matter may occur from the elements other than neutral iron if the direct radiation is blocked and only reflected radiation is visible (Matt et al. 1997, Goldstein et al. 2004). This could be one of the possible reasons for the detection of $\mathrm{Ni} \mathrm{K} \alpha$ in the X-ray spectrum of CD Ind.

A detailed temporal and spectral analyses of the available data will be presented elsewhere (Joshi \& Pandey 2019, in preparation).

\section{Acknowledgements}

This research is based on observations obtained by XMM-Newton, an ESA science mission with instruments and contributions directly funded by ESA Member States and the USA (NASA). We thank the Belgo-Indian Network for Astronomy \& Astrophysics (BINA) approved by the International Division, Department of Science and Technology (DST, Govt. of India; DST/INT/Belg/P-02) and the Belgian Federal Science Policy Office (BELSPO, Govt. of Belgium; BL/11/IN07) for the partial support to visit Royal observatory Belgium for the BINA II workshop.

\section{References}

Aizu K. 1973, PThPh, 49, 1184

Anders E., Grevesse N. 1989, GeCoA, 53, 197

Arnaud K. A. 1996, ASP Conf. Ser., 101, 17

Bernardini F., de Martino D., Mukai K. et al. 2013, MNRAS, 435, 2822

Bowyer S., Lampton M., Lewis J. et al. 1996, ApJS, 102, 129

Boyd D., Patterson J., Allen W. et al. 2014, SASS, 33, 163

Cropper M., Wu K., Ramsay G., Kocabiyik A. 1999, MNRAS, 306, 684

Dorman B., Arnaud K. A. 2001, ASP Conf. Ser., 238, 415

Ferrario L., de Martino D., Gänsicke B. T. 2015, SSRv, 191, 111

Frank J., King A. R., Lasota J.-P. 1988, A\&A, 193, 113

Friedrich S., Staubert R., Lamer G. et al. 1996, A\&A, 306, 860

Goldstein G., Huenemoerder D. P., Blank D. 2004, AJ, 127, 2310

Haberl F., Throstensen J. R., Motch C. et al. 1994, A\&A, 291, 171

Haswell C. A., Patterson J., Thorstensen J. R., Hellier C., Skillman D. R. 1997, ApJ, 476, 847

Jansen F., Lumb D., Altieri B. et al. 2001, A\&A, 365, L1

Joshi A., Pandey J. C., Singh K. P., Agrawal P. C. 2016, ApJ, 830, 56

Joshi A., Pandey J. C., Singh H. P. 2019, AJ, 158, 11

Joshi A., Pandey J. C. 2019, ApJ, in preparation

Katz J. I. 1991, ComAp, 15, 177

Kuijpers J., Pringle J. E. 1982, A\&A, 114, L4 
Lamb D. Q., Masters A. R. 1979, ApJ, 234, L117

Littlefield C., Garnavich P., Mukai K. et al. 2019, ApJ, in press (arXiv:1903.00490)

Mason K. O., Watson M. G., Ponman T. J. et al. 1992, MNRAS, 258, 749

Mason P. A., Ramsay G., Andronov I. et al. 1998, MNRAS, 295, 511

Mason K. O., Breeveld A., Much R. et al. 2001, A\&A, 365, L36

Matt G., Fabian A. C., Reynolds C. S. 1997, MNRAS, 289, 175

Mewe R., Gronenschild E. H. B. M., van den Oord G. H. J. 1985, A\&AS, 62, 197

Mouchet M., Bonnet-Bidaud J. M., Somov N. N., Somova T. A. 1997, A\&A, 324, 109

Myers G., Patterson J., de Miguel E. et al. 2017, PASP, 129, 044204

Norton A. J., Somerscales R. V., Wynn G. A. 2004, ASP Conf. Ser. 315, 216

Ramsay G., Buckley D. A. H., Cropper M., Harrop-Allin M. K. 1999, MNRAS, 303, 96

Ramsay G., Cropper M. 2002a, MNRAS, 334, 805

Rea N., Coti Zelati F., Esposito P. et al. 2017, MNRAS, 471, 2902

Roberts D. H., Lehar J., Dreher J. W. 1987, AJ, 93, 968

Sako M., Liedahl D. A., Kahn S. M., Paerels F. 1999, ApJ, 525, 921

Schwope A. D., Buckley D. A. H., O’Donoghue D. et al. 1997, A\&A, 326, 195

Silber A. D., Szkody P., Hoard D. W. et al. 1997, MNRAS, 290, 25

Smith R. K., Brickhouse N. S., Liedahl D. A., Raymond J. C. 2001, ApJ, 556, L91

Turner M. J. L., Abbey A., Arnaud M. et al. 2001, A\&A, 365, L27

Vennes S., Wickramasinghe D. T., Thorstensen J. R., Christian D. J., Bessell M. S. 1996, AJ, 112, 2254

Voges W., Aschenbach B., Boller T. et al. 1996, IAUC, 6420, 1

Woelk U., Beuermann K. 1992, A\&A, 256, 498

Wu K., Chanmugam G., Shaviv G. 1994, ApJ, 426, 664 